\title{
Pantothenate Kinase Associated Neurodegeneration: A Case Report
}

\author{
Rahar S', Chaudhary A² \\ ${ }^{1}$ Dr. Sanjeev Rahar, MBBS, MS, Senior Resident, Department of Ophthalmology, Safdarjung Hospital and Vardhaman \\ Mahaveer Medical College, New Delhi, India, ${ }^{2}$ Ashok Choudhary, MBBS, MD, Junior Specialist, Department of Paediatrics, \\ Sri Kalyan Hospital, Sikar, Rajasthan, India.
}

Address for correspondence: Dr. Sanjeev Rahar, E-mail: sanjeevrahar@gmail.com

\begin{abstract}
Pantothenate kinase-associated neurodegeneration (PKAN) is a form of neurodegeneration with brain iron accumulation (NBIA), formerly called Hallervorden-Spatz syndrome. PKAN is the first described inborn error of coenzyme A metabolism. PKAN encompasses two clinical subtypes, classic and atypical. We here report an eight year girl diagnosed as classic PKAN. We also review the literature about PKAN.
\end{abstract}

Key words: Eye of tiger sign, NBIA, Neuroregeneration, Iron Accumulation, Hallevorden Spatz Syndrome

\section{Introduction}

P antothenate kinase-associated neurodegeneration (PKAN) is autosomal recessive neurodegenerative disorder. It is the first described inborn error of coenzyme A metabolism. In the absence of genetic diagnostic testing clinical examination and typical MRI finding help in diagnosis of PKAN.

\section{The Case}

An eight year old girl product of third degree consanguineous couple, $2^{\text {nd }}$ in birth order presented with progressive increase in falls while walking and abnormal posturing of limbs for last two years. There was no diurnal variation in symptoms. She also had progressive cognitive decline and decrease in speech output. There was history of decreased vision in dark for past 1 year. Her one elder brother died of similar illness at eleven years of age, whose illness started at the age of eight years. On clinical examination her weight was $15 \mathrm{~kg}$, OFC $51 \mathrm{~cm}$. On clinical examination her vitals were stable, no neurocutaneous markers, no organomegaly. Nervous system examination revealed extrapyramidal dysarthria, decreased speech output, generalized dystonia, spasticity and extensor toe sign. On fundus examination there were bilateral retinal pigmentary changes (Figure 1). Magnetic resonance imaging of brain revealed "eye of tiger" sign (Figure 2.1, 2.2) on T2 weighted images.
On the basis of clinical and radiological findings diagnosis of classical form of pantothenate kinase associated neurodegeneration was made. PANK2 mutation could not be done due to financial constraints. Child was started on trihexyphenidyl, baclofen, physiotherapy, occupational therapy and advised regular follow up.

\section{Discussion}

Pantothenatekinase-associatedneurodegeneration (PKAN) is a form of neurodegeneration with brain iron accumulation (NBIA), formerly called HallervordenSpatz syndrome. Due to Controversy regarding Julius Hallervorden's role in "mercy killings" associated with Nazi activities during World War II has lead recommendations that his name be removed from the name of this condition'. PKAN is inherited in an autosomal recessive manner. PANK2 gene is located on chromosome 20p13. Following the discovery of the PANK2 gene delineated two clinical forms of PKAN, the classic form and an atypical form, based on age at onset and rate of disease progression ${ }^{2}$.

PKAN is the first described inborn error of coenzyme A metabolism. PANK2 protein is targeted to the mitochondria. There is tissue-specific coenzyme A deficiency. Accumulation of cysteine-containing substrates and iron induced lipid peroxidation is responsible in pathogenesis of $\mathrm{PKAN}^{3}$. PKAN is 


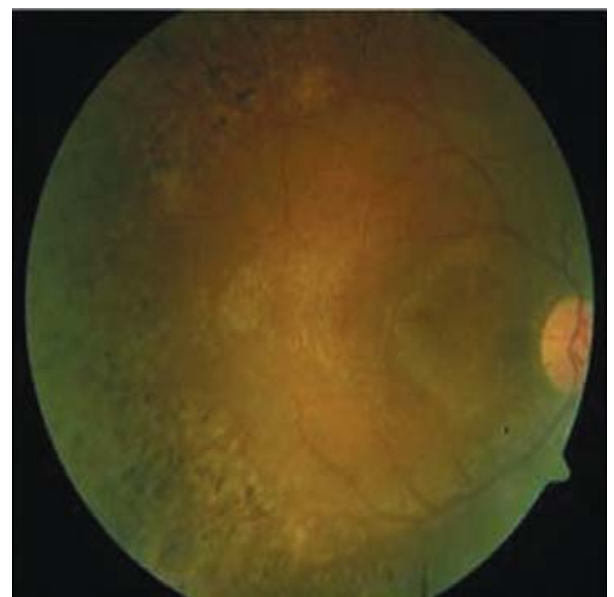

Fig 1: Ophthalmologic finding of right fundus showing retinal pigmentary changes
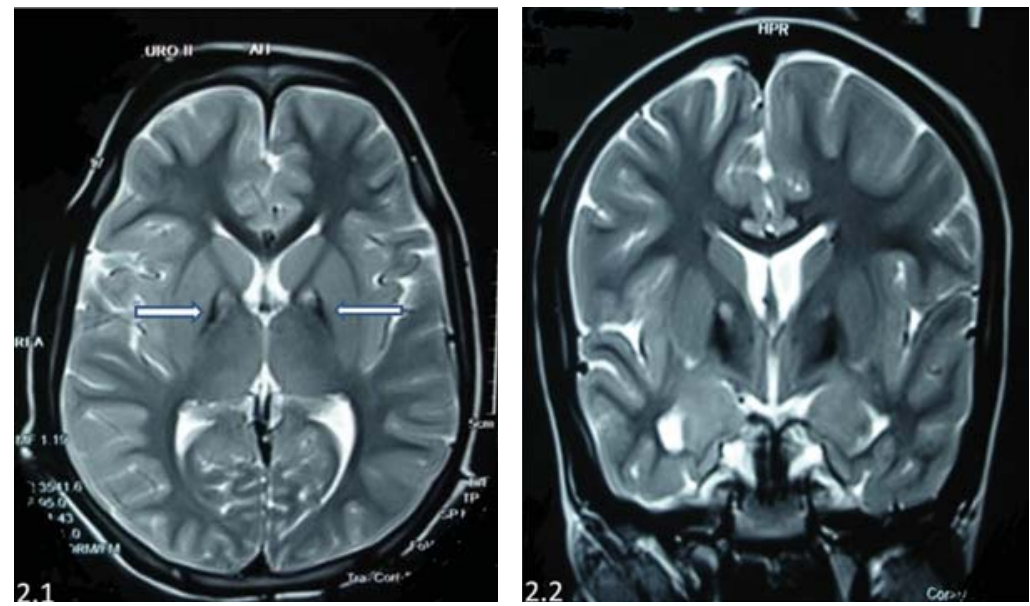

Fig 2: MRI brain showing T2 axial (2.1) and coronal (2.2) images: Bilateral symmetrical hypointensity in globus pallidus with central hyperintensity "eye of tiger sign" (arrows). characterized by progressive dystonia and basal ganglia and substantia nigra iron deposition with onset that usually occurs before age of ten years. Commonly associated features include dysarthria, rigidity, and pigmentary retinopathy. Some of these children (approximately $8 \%$ ) have associated acanthocytosis in their peripheral blood smears. Most of these patients develop difficulties between 5 and 8 years of age. About $25 \%$ of affected individuals have an 'atypical' presentation with later onset (age $>10$ years), prominent speech defects, psychiatric disturbances, and more gradual progression of disease.

Brain magnetic resonance imaging is standard in the diagnostic evaluation of all forms of PKAN. The 'eye of the tiger' sign, a central region of hyperintensity surrounded by a rim of hypointensity on coronal or axial T2-weighted images of the globus pallidus, is highly correlated with the presence of a PANK2 mutation in classic cases ${ }^{4}$.

The hypointense signals on T2 weighted image is due to iron deposition in iron rich brain tissue and the central hyperintensity is due to the glioses and spongioses due to cell death ${ }^{5}$ due to cysteine containing neurotoxic substrates. Areas of the globus pallidus and the pars reticulata of the substantia nigra have proportionately increased iron content in normal individuals; however, in PKAN, iron-containing pigment accumulates to an extraordinary degree in these areas $^{6}$. In classic PKAN, two-thirds of affected individuals demonstrate pigmentary retinopathy.

The eye-of-tiger-sign can sometimes be seen in other NBIA or non NBIA conditions.

In few cases of neuroferritinopathy, corticobasalganglia degeneration and progressive supranuclear palsy esepcially in elderly people with carbon monoxide poisoning eye-of-tiger-sign is seen. However the clinical features like age of onset differentiate these from PKAN. In neuroferritinopathy, MRI reveals involvement of caudate, putamen, thalamus and cerebral cortex ${ }^{7}$. Another condition, where the 'eye of tiger' like appearance is seen, is dihydropteridine reductase deficiency in which there will be associated features like cortical calcifications and cysts. Even the clinical presentation varies with microcephaly, parkinsonism, oculogyric crisis and disturbed psychomotor development ${ }^{8}$.

There is no specific treatment for PKAN. Treatment requires multidisciplinary approach including neurologist, ophthalmologist, physiotherapist, occupational therapist, geneticist and speech therapist. Dystonia is among the most common associated movement abnormalities. Dystonia and spasticity can be managed with Trihexyphenidyl and baclofen. Intrathecal baclofen may be considered if oral treatment fails. Laryngeal dystonia may require tracheotomy if respiration is impaired. Deep brain stimulation (DBS) of globus pallidus may be a effective strategy in PKAN ${ }^{9}$. If there is difficulty in swallowing and nutrition is impaired, gastrostomy placement may prove necessary. Oral glycopyrrolate and hyoscine patch may be useful for drooling. As PKAN is autosomal recessive in inheritance, at each conception sibling of proband has $25 \%$ chance of being affected. Prenatal diagnosis may be possible if facilities of genetic testing are available.

\section{References}

1. Shevell M. Racial hygiene, active euthanasia, and Julius Hallervorden. Neurology 1992;42:2214-9.

2. Zhou B, Westaway SK, Levinson B, Johnson MA, Gitschier J, Hayflick SJ. A novel pantothenate kinase 
gene (PANK2) is defective in Hallervorden-Spatz syndrome. Nat Genet 2001;28:345-9.

3. Gregory A, Hayflick SJ. Folia Neurodegeneration with brain iron accumulation. Neuropathology 2005;43(4):286-96.

4. Hayflick SJ, Westaway SK, Levinson B, Zhou B, Johnson MA, Ching KH, Gitschier J. Genetic, clinical, and radiographic delineation of Hallervorden-Spatz syndrome. N Engl J Med 2003;348:33-40.

5. Adams RJ, Nichols FT, McKie V, McKie K, Milner P, Gammal TE. Hallervorden Spatz syndrome: clinical and magnetic resonance imaging correlations. Ann Neurol 1988;24:692-4.

6. Hallgren B, Sourander P. The effect of age on the non-haemin iron in the human brain. $J$ Neurochem 1958;3:47-51.
7. McNeill A, Birchall D, Hayflick SJ, et al. T2* and FSE MRI distinguishes four subtypes of neurodegeneration with brain iron accumulation. Neurology 2008;70:1614-9.

8. Van der Knaap MS, Valk J. Phenylketonuria. In: Heilmann $U$, editor. Magnetic resonance of myelination and myelin disorders. 3rd ed. Berlin: Springer-Verlag; 2005. p. 284-93.

9. Adamovicová $M$, Jech $R$, Urgošík $D$, Spacková $\mathrm{N}$, Krepelová A. Pallidal stimulation in siblings with pantothenate kinase-associated neurodegeneration: four-year follow-up. Mov Disord 2011;26(1):184.

\section{How to cite this article?}

Rahar S, Chaudhary A. Pantothenate Kinase Associated Neurodegeneration: A Case Report. J Nepal Paediatr Soc 2012;32(1):9092. 